\title{
Deficiency in the double-stranded RNA binding protein HYPONASTIC LEAVES1 increases sensitivity to the endoplasmic reticulum stress inducer tunicamycin in Arabidopsis
}

\author{
Rikako Hirata, Kei-ichiro Mishiba, Nozomu Koizumi and Yuji Iwata* (0)
}

\begin{abstract}
Objective: microRNA (miRNA) is a small non-coding RNA that regulates gene expression by sequence-dependent binding to protein-coding mRNA in eukaryotic cells. In plants, miRNA plays important roles in a plethora of physiological processes, including abiotic and biotic stress responses. The present study was conducted to investigate whether miRNA-mediated regulation is important for the endoplasmic reticulum (ER) stress response in Arabidopsis.

Results: We found that hyl1 mutant plants are more sensitive to tunicamycin, an inhibitor of $\mathrm{N}$-linked glycosylation that causes ER stress than wild-type plants. Other miRNA-related mutants, se and ago 1, exhibited similar sensitivity to the wild-type, indicating that the hypersensitive phenotype is attributable to the loss-of-function of HYL 1 , rather than deficiency in general miRNA biogenesis and function. However, the transcriptional response of select ER stressresponsive genes in hyl1 mutant plants was indistinguishable from that of wild-type plants, suggesting that the lossof-function of HYL1 does not affect the ER stress signaling pathways.
\end{abstract}

Keywords: Arabidopsis thaliana, Endoplasmic reticulum, HYPONASTIC LEAVES1, microRNA, Unfolded protein response

\begin{abstract}
Introduction
microRNA (miRNA) is a non-coding small RNA that regulates gene expression in eukaryotic cells. miRNA binds to mRNA through nucleotide complementarity and mediates mRNA degradation and translation inhibition. The molecular mechanism of miRNA biogenesis has been extensively characterized in both animal and plant systems. In plants, miRNA is transcribed as a precursor RNA, termed primary miRNA (pri-miRNA) and processed by DICER-LIKE1 (DCL1) to generate a miRNA/ miRNA* duplex [1]. One of the strands, miRNA, is incorporated into ARGONAUTE1 (AGO1) to form RNAinduced silencing complex (RISC) to target mRNA to mediate mRNA degradation and translation repression
\end{abstract}

\footnotetext{
*Correspondence: yuji.iwata@plant.osakafu-u.ac.jp

Graduate School of Life and Environmental Sciences, Osaka Prefecture

University, 1-1 Gakuen-cho, Naka-ku, Sakai 599-8531, Osaka, Japan
}

whereas the other strand, miRNA*, is degraded. A number of proteins, such as the double-stranded RNA (dsRNA) binding protein HYPONASTIC LEAVES1 (HYL1) and the zinc finger protein SERRATE (SE), have been reported to function in ensuring accurate and efficient miRNA biogenesis [2-4].

In plants, miRNA plays pivotal roles in a plethora of physiological processes, including abiotic and biotic stress responses and nutrient adaptation [5]. Indeed, a number of stress-related miRNAs have been identified in various plant species. For instance, Arabidopsis has been reported to accumulate miRNAs in response to salinity, drought, and cold [6]. Another example is that a salt-tolerant and a salt-sensitive line of maize express differential miRNA accumulation profiles [7]. miR399, miR395, and miR398 have been shown to be important for phosphate, sulfate, and copper homeostasis in plants [5]. 
The endoplasmic reticulum (ER) is the site of synthesis and maturation of secretory and membrane proteins in eukaryotic cells. ER stress occurs when protein folding and assembly in the ER is perturbed and unfolded and misfolded proteins accumulate in the ER. ER stress triggers a cellular response to maintain homeostasis called the ER stress response or the unfolded protein response (UPR) $[8,9]$. IRE1 is an ER stress sensor widely conserved in eukaryotes. IRE1 is a type-I membrane protein and harbors the ER luminal sensor domain and the cytosolic kinase and ribonuclease domains [10]. In plants, IRE1 mediates transcriptional activation of genes encoding ER chaperones and folding enzymes, including BINDING PROTEIN (BiP), through cytoplasmic splicing of bZIP60 mRNA, which produces an active transcription factor $[11,12]$. IRE1 also mediates degradation of mRNA encoding secretory and membrane proteins to reduce the load of newly synthesized proteins into the stressed ER [13].

Although regulation of gene expression by miRNA is involved in a number of stress responses in plants, its involvement in the ER stress response has not been investigated. The present study was conducted to investigate a possible involvement of miRNA-related proteins in the ER stress response in Arabidopsis.

\section{Main text \\ Methods \\ Plant materials}

We used Arabidopsis thaliana Col-0 as wild-type and previously reported mutants in Col-0 background, hyl12 [4], se-1 [14], ago1-46 [15], and irelab [11]. HYL1YFP/hyl1-2 is hyl1-2 mutant expressing HYL1-YFP fusion proteins under $H Y L 1$ promoter [16].

\section{Sensitivity assay to ER stress-inducing agents}

The sensitivity assay was carried out as previously described [17]. Briefly, Arabidopsis seeds of indicated genotypes were surface sterilized and sown on halfstrength Murashige Skoog (MS) medium containing 1\% sucrose supplemented with indicated concentrations of tunicamycin or $0.1 \%$ dimethyl sulfoxide (DMSO) as a solvent control. Sterilized seeds were grown under $16 \mathrm{~h}$-light $/ 8 \mathrm{~h}$-dark cycle at $23^{\circ} \mathrm{C}$ for 10 days and photographed. Seedlings with open cotyledons were counted as survived. Error bars represent standard errors calculated from three biological replicates.

\section{Quantitative reverse transcription-PCR ( $q R T-P C R)$}

Ten-day-old wild-type and mutant seedlings grown in half-strength MS medium containing $1 \%$ sucrose were treated with $5 \mu \mathrm{g} / \mathrm{mL}$ tunicamycin for indicated time periods and ground by using a mortar and pestle with liquid nitrogen. Total RNA was extracted using acid guanidinium thiocyanate, phenol, and chloroform as described elsewhere [18].

One hundred ng RNA was reverse transcribed by High-Capacity cDNA Reverse Transcription Kit (Applied Biosystems) using random hexamer according to the manufacturer's instruction. Quantitative real-time PCR was performed by using Applied Biosystems 7300 RealTime PCR Systems (Applied Biosystems) with THUNDERBIRD SYBR qPCR Mix (TOYOBO) according to the manufacturer's instruction. Primers used were listed in Additional file 1. Error bars represent standard errors calculated from three biological replicates.

\section{Results}

\section{hyl1 mutant is more sensitive to ER stress}

We first asked whether miRNA-related Arabidopsis mutants exhibit altered ER stress sensitivity. We examined hyl1-2, se-1, and ago1-46 mutants for ER stress sensitivity. As shown in Fig. 1a, compared to wild-type seedlings, growth of hyl1-2 mutant seedlings was more severely affected by treatment with tunicamycin, an inhibitor of $\mathrm{N}$-linked glycosylation, which disrupts folding of glycosylated proteins, thereby causing ER stress. In contrast, growth of se-1 and ago 1-46 mutant seedlings was similar to that of the wild-type seedlings. As shown in Fig. 1b, the survival rate was also significantly affected in hyl1-2 mutant, but not in se-1 and ago1-46 mutants, when grown on tunicamycin-containing MS medium. The observation that only hyl1-2 mutant was more sensitive to tunicamycin suggests that the hypersensitive phenotype of hyl1-2 mutant to tunicamycin is attributed to the deficiency in the function of HYL1, rather than the defective miRNA biogenesis.

This hypersensitive phenotype of the hyl1-2 mutant was complemented by expressing the HYL1-YFP fusion construct (Fig. 1c, d), confirming that the disruption of HYL1 accounts for the observed tunicamycin hypersensitivity.

\section{A transcriptional response of hyl1 mutant plants appears to be indistinguishable from that of wild-type plants}

We next compared the sensitivity of hyl1-2 mutant to that of ire1ab, a known mutant that exhibit hypersensitivity to ER stress due to the defective transcriptional response to ER stress [11]. As shown in Fig. 2a, the hypersensitivity of hyl1-2 mutant to tunicamycin was comparable to that of ire $1 a b$ mutant.

We then asked whether hyl1-2 mutant is defective in the UPR. We treated wild-type and hyl1-2 and ire $1 a b$ mutant seedlings with tunicamycin and subjected them to qRT-PCR analysis. We first detected BiP3 as a gene induced in an IRE1-dependent manner in the early phase of the UPR $[19,20]$. As shown in Fig. 2b, BiP3 


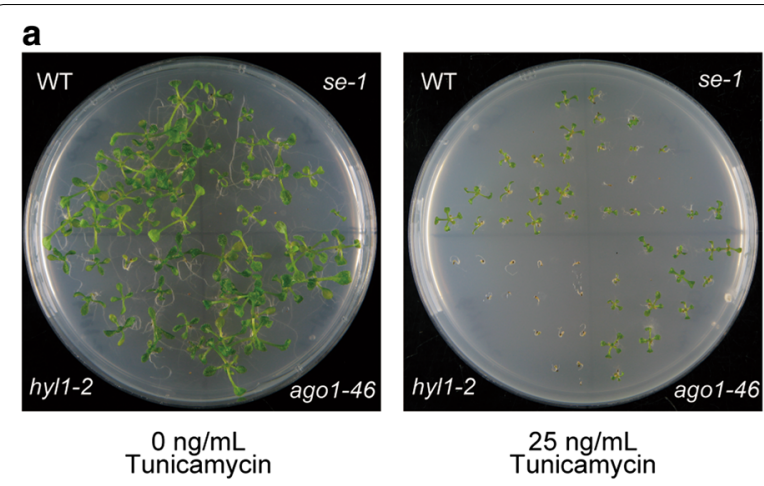

b

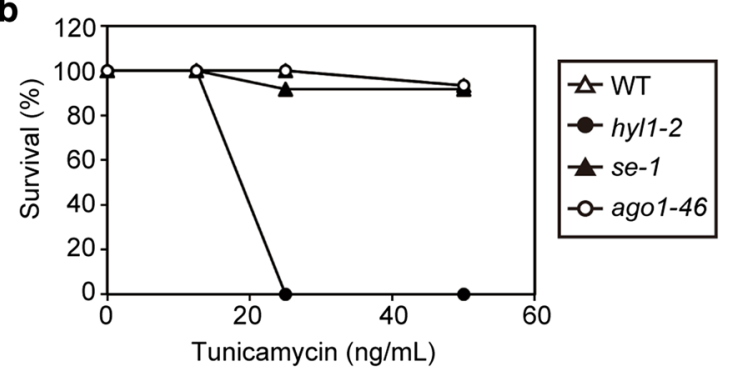

C

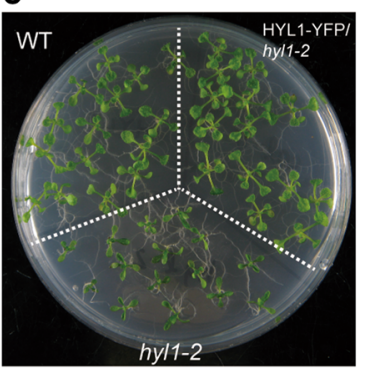

$0 \mathrm{ng} / \mathrm{mL}$

Tunicamycin

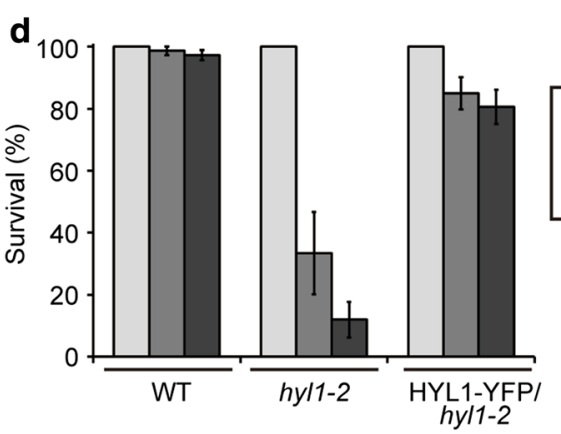

Fig. 1 Sensitivity of Arabidopsis miRNA-related mutants to ER stress. a Sensitivity of miRNA-related mutants to tunicamycin. Seeds of wild-type (WT), hyl1-2, se-1, and ago 1-46 were sown on half-strength MS medium with indicated concentrations of tunicamycin, grown for 10 days, and photographed. $\mathbf{b}$ Effect of tunicamycin on survival of miRNA-related mutants. Seeds of indicated genotypes were grown for 10 days as in a, and the percentage of seeds that survived was calculated. c Sensitivity of hy/1-2 and HYL1-YFP/hy/1-2 plants to tunicamycin. Seedlings of wild-type (WT), hy/1-2, and HYL1-YFP/hy/1-2 were grown as in $\mathbf{a}$ and photographed. $\mathbf{d}$ Effect of tunicamycin on survival of hyl1-2 and HYL1-YFP/hyl1-2 plants. Seeds of indicated genotypes were grown for 10 days as in $\mathbf{c}$, and the percentage of seeds that survived was calculated was upregulated in hyl1-2 mutant as well as in wildtype seedlings, whereas irelab mutant seedlings showed compromised BiP3 induction as previously reported [11]. We next detected another ER stress-responsive gene, At5g40010. At5g40010 is a gene up-regulated to some extent in the wild-type but more strongly in ire1ab mutant, presumably because ire $1 a b$ mutant undergoes more severe cell damage [13]. As shown in Fig. 2b, At5g40010 transcripts responded similarly to tunicamycin in hyl1-2 mutant as well as in the wild-type, whereas ire $1 a b$ mutant exhibited stronger At5g40010 induction as previously reported [13]. Other genes that encode ERresident molecular chaperones and folding enzymes also exhibit similar expression both in the wild-type and hyl12 mutant plants (Fig. 2c). We also tested se-1 and ago1-46 mutants, which exhibit similar tunicamycin sensitivity to the wild-type plants, for two ER chaperone genes; IRE1dependent BiP3 and IRE1-independent CRT2. As shown in Fig. 2d, induction of these two genes in both se-1 and ago1-46 mutants was similar to that in the wild-type (Fig. 2d). Taken together, the transcriptional response of hyl1-2, se-1, and ago1-46 mutant plants is indistinguishable from that of wild-type plants.

\section{Discussion}

We demonstrated in the present study that, among Arabidopsis miRNA-related mutants tested, only hyl1-2 mutant was more sensitive to the ER stress inducer tunicamycin while se-1 and ago1-46 mutants did not exhibit such hypersensitivity. It suggests that an overall reduction in miRNA biogenesis and function is not the cause of the observed tunicamycin oversensitivity.

One possible interpretation is that miRNAs that show less accumulation specifically in hyl1-2 mutant accounts for the observed hypersensitivity. Indeed, not all miRNAs are similarly affected among miRNA-related mutants [21], and the observable growth and morphological phenotypes of those mutants were not identical. Therefore, it is plausible that less accumulation of some of miRNAs whose accumulation is more dependent on HYL1 stabilizes their target mRNAs, resulting in ER stress sensitivity.

Another possible interpretation is that HYL1-specific function contributes to ER stress tolerance. In plants, miRNA biogenesis, in which pri-miRNA transcripts are processed by DCL1 to generate mature miRNA, occurs in the subnuclear bodies called D-bodies in the nucleus. HYL1 is a dsRNA-binding protein that increases primiRNA processing by DCL1 [1]. Although HYL1 is primarily localized in D-bodies and nucleoplasm in the nucleus, a subfraction of HYL1 is also detected in the cytoplasm [22]. Therefore, it is conceivable that 

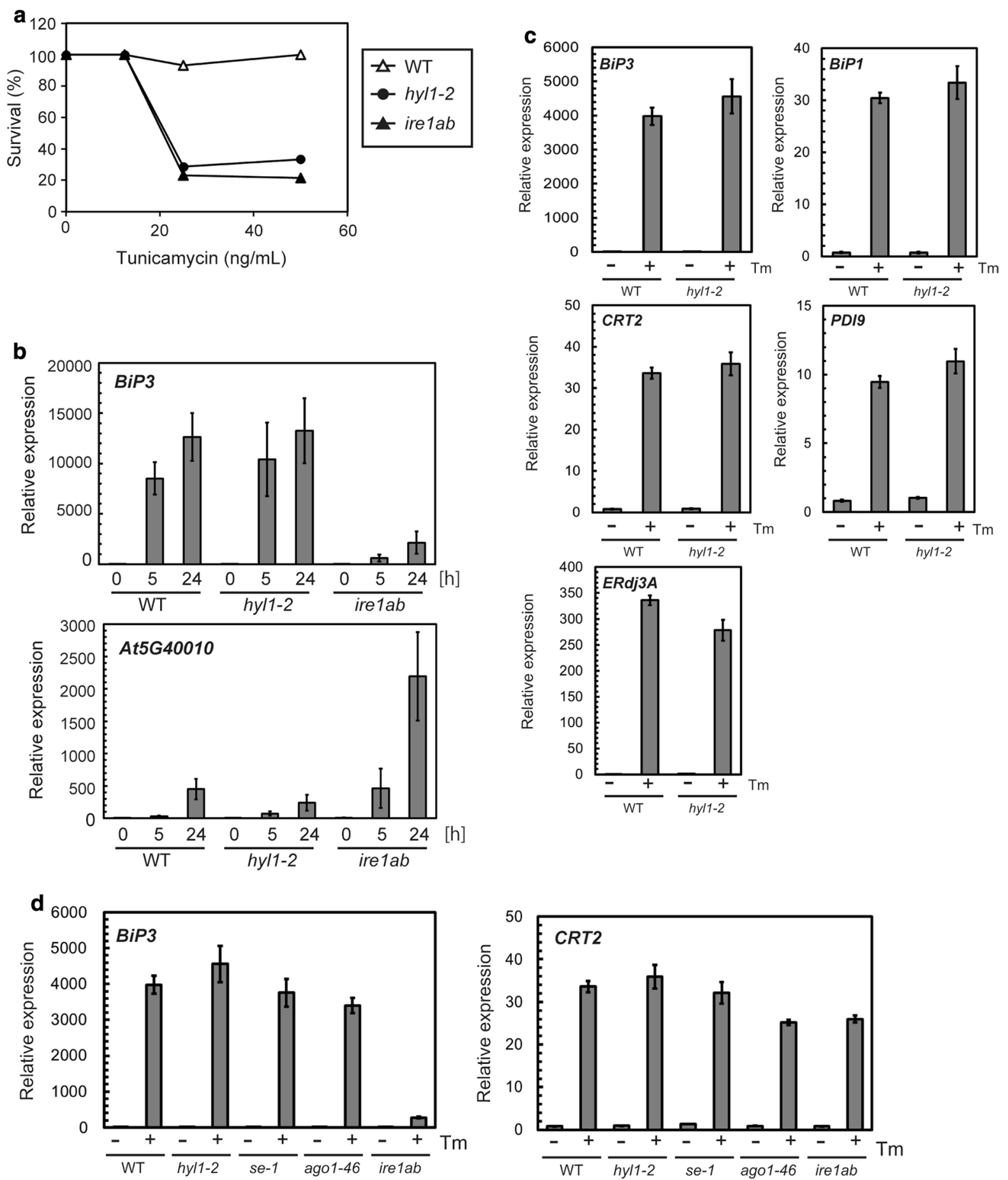

Fig. 2 Sensitivity and transcriptional response of hyl1-2 and ire $1 a b$ mutants to tunicamycin. a Effect of tunicamycin on survival of wild-type, hyl1-2, and ire 1ab. Seeds of wild-type (WT), hyl1-2, and ire 1ab were sown on half-strength MS medium with indicated concentrations of tunicamycin and grown for 10 days. The percentage of seeds that survived was calculated. $\mathbf{b}$ qRT-PCR analysis of wild-type and hy/1-2 and ire $1 a b$ mutant seedlings to tunicamycin. Ten-day-old wild-type and hy/1-2 and ire $1 a b$ mutant seedlings were treated with $5 \mu \mathrm{g} / \mathrm{mL}$ tunicamycin for indicated times and subjected to qRT-PCR analysis for detecting BiP3 and At5940010. The expression values of indicated genes were normalized to that of Act8. c qRT-PCR analysis of wild-type and hy/1-2 mutant seedlings to tunicamycin. Ten-day-old wild-type and hyl1-2 mutant seedlings were treated with $5 \mathrm{\mu g} / \mathrm{mL}$ tunicamycin $(+\mathrm{Tm})$ or $0.1 \%$ DMSO $(-\mathrm{Tm})$ as a solvent control for $5 \mathrm{~h}$, and subjected to qRT-PCR analysis for detecting indicated genes. The expression values of indicated genes were normalized to that of Act8. $\mathbf{d}$ qRT-PCR analysis of wild-type and hyl1-2, se-1, and ago 1-46 mutant seedlings to tunicamycin. Ten-day-old wild-type and mutant seedlings were treated with tunicamycin or DMSO and subjected to qRT-PCR analysis for BiP3 and CRT2 as in c 
cytoplasmically localized HYL1 plays a role in recovering cellular damages caused by ER stress.

There have been recent reports that implicate a link between miRNA function and the ER. It has been reported that AGO1 is a peripheral ER membrane protein and that miRNA-mediated translational repression occurs on the ER membrane in plants [23]. Furthermore, in animal systems, IRE1 has been reported to destabilize a subset of pre-miRNAs to reduce accumulation of select miRNAs and initiate apoptosis, demonstrating the involvement of the UPR signaling pathway component in miRNA biogenesis [24]. Those reports prompted us to speculate a possible connection between miRNA biogenesis and function machineries and the ER stress response. However, the present study was unable to identify such connections, because hyl12 mutant exhibited a normal transcriptional response to ER stress despite its significantly less tolerance. Nevertheless, this study presents an interesting observation that one of miRNA biogenesis-related mutants exhibits hypersensitivity to ER stress-inducing agents. Further analyses such as genome-wide transcript profiling would be required to elucidate the role of HYL1 in ER stress tolerance and the UPR.

\section{Limitations}

The shortcoming of this paper is that the function of HYL1 during the ER stress response remains to be elucidated due to the lack of further experimental analyses such as transcriptome profiling using RNA-seq.

\section{Supplementary information}

Supplementary information accompanies this paper at https://doi. org/10.1186/s13104-019-4623-3.

Additional file 1: Primers used for qRT-PCR.

\section{Abbreviations}

AGO1: ARGONAUTE1; BiP: BINDING PROTEIN; DCL1: DICER-LIKE1; DMSO: dimethyl sulfoxide; dsRNA: double-stranded RNA; ER: endoplasmic reticulum; HYL1: HYPONASTIC LEAVES1; miRNA: microRNA; MS: Murashige and Skoog; pri-miRNA: primary miRNA; qRT-PCR: quantitative reverse transcription PCR; SE: SERRATE; UPR: unfolded protein response.

\section{Acknowledgements}

We thank Dr. Yuda Fang (Shanghai Institute for Biologcal Sciences) for providing HYL1-YFP/hyl1-2 seeds.

\section{Authors' contributions}

$\mathrm{RH}$ and $\mathrm{YI}$ designed the experiments. $\mathrm{RH}$ and $\mathrm{YI}$ performed the experiments and analyzed the data. $\mathrm{RH}, \mathrm{KM}, \mathrm{NK}$, and $\mathrm{YI}$ wrote the manuscript. All authors read and approved the final manuscript.

\section{Funding}

This work was supported by JSPS KAKENHI Grant Number JP17K15416 to Y.I. The funding body had no role in the experimental design of the study, the collection, analysis and interpretation of data, or the writing of the manuscript.

\section{Availability of data and materials}

The data obtained and analysed during the current study are available from the corresponding author on reasonable request.

Ethics approval and consent to participate

Not applicable.

\section{Consent for publication}

Not applicable.

\section{Competing interests}

The authors declare that they have no competing interests.

Received: 28 February 2019 Accepted: 9 September 2019

Published online: 14 September 2019

\section{References}

1. Voinnet O. Origin, biogenesis, and activity of plant microRNAs. Cell. 2009;136:669-87. https://doi.org/10.1016/j.cell.2009.01.046.

2. Iwata Y, Takahashi M, Fedoroff NV, Hamdan SM. Dissecting the interactions of SERRATE with RNA and DICER-LIKE 1 in Arabidopsis microRNA precursor processing. Nucleic Acids Res. 2013;41:9129-40. https://doi. org/10.1093/nar/gkt667.

3. Dong Z, Han M-H, Fedoroff N. The RNA-binding proteins HYL1 and SE promote accurate in vitro processing of pri-miRNA by DCL1. Proc Natl Acad Sci. 2008;105:9970-5. https://doi.org/10.1073/pnas.0803356105.

4. Vazquez F, Gasciolli V, Crété P, Vaucheret $\mathrm{H}$. The nuclear dsRNA binding protein HYL1 is required for microRNA accumulation and plant development, but not posttranscriptional transgene silencing. Curr Biol. 2004;14:346-51. https://doi.org/10.1016/j.cub.2004.01.035.

5. Khraiwesh B, Zhu J-K, Zhu J. Role of miRNAs and siRNAs in biotic and abiotic stress responses of plants. Biochim Biophys Acta. 2012;1819:137-48. https://doi.org/10.1016/j.bbagrm.2011.05.001.

6. Liu H-H, Tian X, Li Y-J, Wu C-A, Zheng C-C. Microarray-based analysis of stress-regulated microRNAs in Arabidopsis thaliana. RNA. 2008;14:836-43. https://doi.org/10.1261/rna.895308.

7. Ding D, Zhang L, Wang H, Liu Z, Zhang Z, Zheng Y. Differential expression of miRNAs in response to salt stress in maize roots. Ann Bot. 2009;103:2938. https://doi.org/10.1093/aob/mcn205.

8. Ron D, Walter P. Signal integration in the endoplasmic reticulum unfolded protein response. Nat Rev Mol Cell Biol. 2007;8:519-29. https://doi. org/10.1038/nrm2199.

9. Walter $P$, Ron D. The unfolded protein response: from stress pathway to homeostatic regulation. Science. 2011;334:1081-6. https://doi. org/10.1126/science.1209038.

10. Iwata Y, Koizumi N. Plant transducers of the endoplasmic reticulum unfolded protein response. Trends Plant Sci. 2012;17:720-7. https://doi. org/10.1016/j.tplants.2012.06.014.

11. Nagashima Y, Mishiba K-I, Suzuki E, Shimada Y, Iwata Y, Koizumi N. Arabidopsis IRE1 catalyses unconventional splicing of bZIP60 mRNA to produce the active transcription factor. Sci Rep. 2011;1:29. https://doi. org/10.1038/srep00029.

12. Deng Y, Humbert S, Liu J-X, Srivastava R, Rothstein SJ, Howell SH. Heat induces the splicing by IRE1 of a mRNA encoding a transcription factor involved in the unfolded protein response in Arabidopsis. Proc Natl Acad Sci USA. 2011;108:7247-52. https://doi.org/10.1073/pnas.1102117108.

13. Mishiba K, Nagashima Y, Suzuki E, Hayashi N, Ogata Y, Shimada Y, et al. Defects in IRE1 enhance cell death and fail to degrade mRNAs encoding secretory pathway proteins in the Arabidopsis unfolded protein response. Proc Natl Acad Sci USA. 2013;110:5713-8. https://doi.org/10.1073/ pnas. 1219047110.

14. Prigge MJ, Wagner DR. The arabidopsis serrate gene encodes a zincfinger protein required for normal shoot development. Plant Cell. 2001;13:1263-79. https://doi.org/10.1105/tpc.010095.

15. Smith MR, Willmann MR, Wu G, Berardini TZ, Moller B, Weijers D, et al. Cyclophilin 40 is required for microRNA activity in Arabidopsis. Proc Natl Acad Sci. 2009;106:5424-9. https://doi.org/10.1073/pnas.0812729106.

16. Liu Q, Yan Q, Liu Y, Hong F, Sun Z, Shi L, et al. Complementation of HYPONASTIC LEAVES1 by double-strand rna-binding domains of DICER-LIKE1 
in nuclear dicing bodies. Plant Physiol. 2013;163:108-17. https://doi org/10.1104/pp.113.219071.

17. Iwata Y, Ashida M, Hasegawa C, Tabara K, Mishiba K, Koizumi N. Activation of the Arabidopsis membrane-bound transcription factor bZIP28 is mediated by site-2 protease, but not site-1 protease. Plant J. 2017;91:408-15. https://doi.org/10.1111/tpj.13572.

18. Chomczynski P, Sacchi N. The single-step method of RNA isolation by acid guanidinium thiocyanate-phenol-chloroform extraction: twentysomething years on. Nat Protoc. 2006;1:581-5. https://doi.org/10.1038/ nprot.2006.83.

19. Iwata Y, Sakiyama M, Lee M-H, Koizumi N. Transcriptomic response of Arabidopsis thaliana to tunicamycin-induced endoplasmic reticulum stress. Plant Biotechnol. 2010;27:161-71. https://doi.org/10.5511/plant biotechnology.27.161.

20. Iwata Y, Fedoroff N, Koizumi N. Arabidopsis bZIP60 is a proteolysisactivated transcription factor involved in the endoplasmic reticulum stress response. Plant Cell. 2008;20:3107-20. https://doi.org/10.1105/ tpc.108.061002.

21. Manavella PA, Hagmann J, Ott F, Laubinger S, Franz M, Macek B, et al. Fast-forward genetics identifies plant CPL phosphatases as regulators of miRNA processing factor HYL1. Cell. 2012;151:859-70. https://doi. org/10.1016/j.cell.2012.09.039.

22. Zhang Z, Guo X, Ge C, Ma Z, Jiang M, Li T, et al. KETCH1 imports HYL1 to nucleus for miRNA biogenesis in Arabidopsis. Proc Natl Acad Sci. 2017;114:4011-6. https://doi.org/10.1073/pnas.1619755114.

23. Li S, Liu L, Zhuang X, Yu Y, Liu X, Cui X, et al. MicroRNAs inhibit the translation of target mRNAs on the endoplasmic reticulum in Arabidopsis. Cell. 2013;153:562-74. https://doi.org/10.1016/j.cell.2013.04.005.

24. Upton J-P, Wang L, Han D, Wang ES, Huskey NE, Lim L, et al. IRE1 cleaves select microRNAs during ER stress to derepress translation of proapoptotic caspase-2. Science. 2012;338:818-22. https://doi.org/10.1126/scien ce.1226191.

\section{Publisher's Note}

Springer Nature remains neutral with regard to jurisdictional claims in published maps and institutional affiliations.
Ready to submit your research? Choose BMC and benefit from:

- fast, convenient online submission

- thorough peer review by experienced researchers in your field

- rapid publication on acceptance

- support for research data, including large and complex data types

- gold Open Access which fosters wider collaboration and increased citations

- maximum visibility for your research: over $100 \mathrm{M}$ website views per year

At BMC, research is always in progress.

Learn more biomedcentral.com/submissions 